\title{
AN ENHANCED RUN-LENGTH ENCODING COMPRESSION METHOD FOR TELEMETRY DATA
}

\author{
Yanhu Shan ${ }^{1,2)}$, Yongfeng Ren ${ }^{1,2)}$, Guoyong Zhen ${ }^{1,2)}$, Kaiqun Wang ${ }^{3)}$ \\ 1) North University of China, National Key Laboratory For Electronic Measurement Technology, Taiyuan 030051, China \\ ( shanyanhu@nuc.edu.cn, +86351392 3640) \\ 2) North University of China, Key Laboratory of Instrumentation Science \& Dynamic Measurement, Ministry of Education, \\ Taiyuan 030051, China (renyongfeng@nuc.edu.cn, zengguoyong@nuc.edu.cn) \\ 3) Taiyuan University of Technology, College of Mechanics, Taiyuan 030024, China (wangkaiqun@tyut.edu.cn)
}

\begin{abstract}
The telemetry data are essential in evaluating the performance of aircraft and diagnosing its failures. This work combines the oversampling technology with the run-length encoding compression algorithm with an error factor to further enhance the compression performance of telemetry data in a multichannel acquisition system. Compression of telemetry data is carried out with the use of FPGAs. In the experiments there are used pulse signals and vibration signals. The proposed method is compared with two existing methods. The experimental results indicate that the compression ratio, precision, and distortion degree of the telemetry data are improved significantly compared with those obtained by the existing methods. The implementation and measurement of the proposed telemetry data compression method show its effectiveness when used in a high-precision high-capacity multichannel acquisition system.
\end{abstract}

Keywords: multichannel acquisition system, high compression performance, run-length encoding with error parameter, oversampling, hardware implementation.

(C) 2017 Polish Academy of Sciences. All rights reserved

\section{Introduction}

A telemetry testing system is used to evaluate the performance of aircraft and to diagnose failures by detecting the aircraft responses to different input sensor signals in flight experiments. The testing signals are either transmitted to the ground test station by a wireless information channel of the telemetry system or stored in a recorder whose contents will be recycled after the experiment. A highly efficient information channel transmission and telemetry data storage are essential because of an increasing quantity of testing data and their types, as well as high real-time testing requirements [1-3]. The compression of telemetry data will be further used to optimize the bandwidth usage rate of signal channels and storage space.

Many data compression techniques have been employed in the transmission and storage of telemetry data. The most known compression methods include: the run-length encoding (RLE), Huffman encoding, Golomb-Rice encoding, Lempel-Ziv-Welch (LZW), and wavelet compression [4-7]. The telemetry data are obtained from sensors in a form of text, images, video, audio, and many other formats. Heterogeneous types of telemetry data may need various applications of data compression techniques [8]. Maluf et al. combined discrete Fourier transforms (DFTs) with LZW and Flate algorithms for textual data and JPEG coding for images [8]. Two almost lossless data compression algorithms were proposed for the telemetry data produced by hyperspectral sensors installed on a satellite [9]. The telemetry video signals of the surface of Mars are compressed with a compression ratio of 24:1 and then transmitted from the Mars to the Earth by USA Mars Exploration Rovers [10]. A two-stage Lempel-Ziv lossless data compression is implemented for the telemetry data from various sensors aboard satellite launch 
vehicles [11]. A modified Rice compression method is adopted to the telemetry data of a launch vehicle with a compression ratio of 2:1 [12]. Combining multiple data compression algorithms facilitates obtaining a high compression ratio [13-15]. Furthermore, proper hardware implementation of data compression is essential in enhancing the performance of data compression [16, 17]. Kao et al. developed a modularized approach to trade off the hardware cost and an achievable compression ratio [18]. Lin et al. proposed a two-stage hardware architecture to improve compression and decompression rates [4].

The precision, distortion degree, and compression ratio of the telemetry data should be considered. The ideal condition is one with a high compression ratio, high data precision, and no signal distortion. For practical engineering applications, specific values of these parameters should be adjusted to a specific requirement. The compression distortion degree is generally set to less than $0.1 \%$ for engineering applications. A range of compression ratio of the telemetry data in the previous research is from 1.38 to $42[9,19,20]$. The data precision is larger than 8 bits.

Despite the progress in data compression studies, the telemetry data compression used in telemetry acquisition systems has not been sufficiently examined yet. Firstly, given large amounts of the telemetry data and high real-time testing requirements, a data compression method ensuring a high compression ratio is required. Secondly, an effective hardware implementation approach to the data compression method is needed for a real multichannel acquisition system. Many compression methods can obtain a high compression ratio by employing a complex hardware support, such as DSP and FPGA groups or a dedicated compressor [21, 22]. However, these types of hardware implementation are unsuitable for some telemetry engineering applications because of their high cost and complex design. Thirdly, the telemetry signals collected by a telemetry system operating with high sampling rates include interference signals mixed with useful signals because of the harsh environment. So, telemetry signal noise has a negative effect on the data compression ratio.

To address the aforementioned challenges, we propose an effective data compression method based on a combination of the oversampling technology and the RLE compression algorithm with an error factor. In the study there are considered mainly the telemetry data. The proposed approach consists of three modularized phases: 1) oversampling and normal averaging; 2) data encoding based on RLE with an error factor; and 3) two-stage framing. The hardware implementation of the modularized compression method for the telemetry data employs FPGAs. The proposed data compression method used in a telemetry testing system demonstrates the following unique advantages in comparison with the existing data compression methods.

Firstly, the proposed method exhibits a high performance in handling the telemetry data. The previous work enhanced a compression ratio by increasing the error parameter [23]. However, it resulted in a significant increase of a signal distortion and a significant reduction of the acquisition system precision. To address this issue, our approach considers all three factors by incorporating the oversampling technology into the RLE algorithm with a compressibility error. The proposed method achieves a high compression ratio and improves accuracy of the telemetry data for a small error parameter value with a low signal distortion. The proposed method can be also effectively applied to high-precision multichannel acquisition systems.

Secondly, the hardware implementation of the proposed compression method is based on FPGAs. Compressing large amounts of the telemetry data, especially high-precision data, is not feasible without a proper hardware support [24]. However, an effective hardware implementation for telemetry data compression is still unavailable. In our approach, the data compression method employs FPGAs to achieve modularized compression in a multichannel acquisition system. The developed hardware implementation is suitable for the telemetry data because of its low cost and simple design. 


\section{Design and implementation of telemetry data compression method}

The telemetry data used in our study mainly come from various types of sensor signals in aircraft. The telemetry data come in a form of text, images, video, audio, and many other formats. In our work, there is considered mainly the compression method for numerical data, and the telemetry data are transformed into a digital form. Digital signals from each channel of the sensor network in a multichannel telemetry acquisition system are assembled in a frame processor by a quantization processor. The signals are later transmitted to a transmitter or storage system through a sending processor being a unit of the frame processor. This process demonstrates a typical transmission path of the telemetry signals. However, the telemetry signals collected by the telemetry system operating with high sampling rates consist of useful signals mixed with interference signals. The signals are preprocessed before compression to minimize a negative effect of noise on data compression. To this purpose, an improved approach is proposed, which consists of three modularized phases: 1) oversampling and normal averaging of the telemetry data; 2) data compression based on RLE with an error parameter; and 3) two-stage framing. The improved transmission path is shown in Fig. 1.

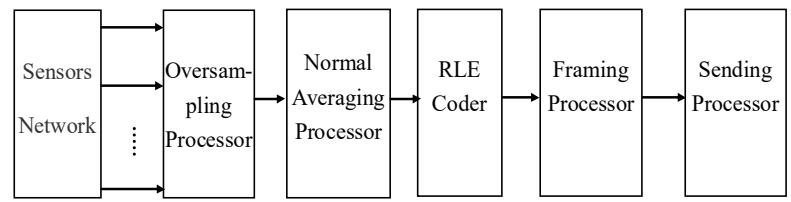

Fig. 1. Transmission patch of the telemetry signal.

\subsection{Telemetry data pre-processing}

To reduce the negative effect of noise on data compression, the telemetry data are preprocessed using oversampling and averaging technologies before data compression. The oversampling prevents aliasing, improves resolution, and reduces noise. Sampling of telemetry signals is based on the Nyquist theory. An anti-aliasing filter is commonly adopted in the acquisition circuit to significantly inhibit signal aliasing. However, full elimination of aliasing by using a practical filter is difficult. The oversampling technology could further improve the effectiveness of inhibition of signal aliasing according to the principle of Nyquist theory. Moreover, the oversampling has a significant influence on the baseband quantization noise when the ADC noise could be approximated as white noise [25-27]. An oversampling frequency $f_{O S}$ of each channel and an oversampling factor $\beta$ are defined as follows:

where $f_{N S}$ is an original sampling frequency.

$$
f_{O S}=\beta f_{N S}
$$

$$
\beta=4^{w} \text {, }
$$

where $w$ is the number of additional bits of a desired resolution.

A quantization noise power $Q_{O S}$ is expressed as:

$$
Q_{O S}=\frac{Q_{N} \times f_{N S}}{f_{O S}} .
$$

Equation (1) is substituted into (3) to obtain the quantization noise power at the oversampling frequency:

$$
Q_{O S}=\frac{q^{2} \times f_{N S}}{12\left(\beta f_{N S}\right)}=\frac{Q_{N}}{\beta} .
$$

Therefore, the signal-to-quantization-noise ratio (SQNR) is further enhanced: 


$$
S Q N R=\frac{Q_{\text {signal }}}{Q_{O S}}
$$

We assume that $N$ is the number of bits without oversampling. Therefore, in our proposed approach, the resolution is equal to the effective number of bits $(N+w)$. We can find that oversampling reduces the quantization noise power and hence increases the resolution of an $\mathrm{N}$ bit ADC and improves SQNR.

The total sampling frequency of the system, $f_{t}$, is determined by the oversampling frequency fos of every input signal and the number $m$ of input channels in the acquisition system, and is denoted as follows:

$$
m \times f_{O S} \leq f_{t} .
$$

The working mode of ADC module is controlled by FPGA by adjusting the total sampling frequency of the system.

The oversampled data are averaged according to the following procedure. To reduce the complexity of sample logic, four nodes (e.g. $A\left(t_{0}\right), A\left(t_{1}\right), A\left(t_{2}\right)$, and $\left.A\left(t_{3}\right)\right)$ are sampled in one sampling cycle. The four sampling points are averaged by the averaging processor to reduce the negative effect of noise on data compression. The averaged data $A\left(T_{1}\right)$ are written into the compression register of a current channel. This procedure is repeated until the signals of all channels are processed. All averaged data (e.g. $\left.A\left(T_{1}\right), A\left(T_{2}\right), \ldots, A\left(T_{n}\right)\right)$ of the corresponding channels are later compressed. A schematic of sampling and averaging is shown in Fig. 2. The interference signals are highly suppressed by the oversampling and averaging processes, which enable to obtain a high compression ratio.

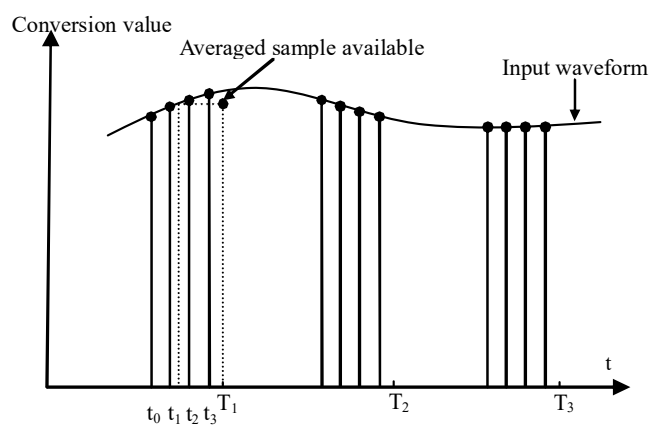

Fig. 2. Sampling and averaging process.

\subsection{Data encoding based on RLE with error factor}

The RLE algorithm is suitable for compressing binary sequences [28]. Therefore, the RLE algorithm is adopted to directly compress signals from each channel of the multichannel acquisition system. The amplitude of all telemetry data are adjusted into the same allowable amplitude range of ADC by a signal adjustment circuit. Subsequently, the adjusted data are transformed into the corresponding quantized data according to bits of ADC. The error parameter is incorporated into the RLE algorithm to increase the compression efficiency. The error bound is related with these quantized data and is set to a specific value on the basis of the distortion tolerance. The differences between adjacent quantized data are compared with the error bound. These quantized data are regarded as being of the same value if their difference is smaller than the error bound; otherwise they are of different values. Therefore, the error bound is irrelevant to the input analog signals, whether they are preprocessed or not.

In our multichannel acquisition system, the resolution of $\mathrm{AD}$ converter was set to 16 bits. The data formats are shown in Table 1 . The highest bit, D15, is defined as a logic number (one 
or zero) to distinguish the quantization data from the run length. Thus, D14 to D0 denote the $\mathrm{AD}$ quantization data if $\mathrm{D} 15$ is equal to one; otherwise, they denote the run length. The averaged data were compressed based on the run-length compression with an error parameter. A flow chart of the compression algorithm process is shown in Fig. 3. Taking channel one as an example, the previous data and the current data were first allocated to the initial values of registers D0 and D1. Secondly, D1 can be replaced by D0 and Nt adds one if D1 is in an interval of $[\mathrm{D} 0-\Delta, \mathrm{D} 0+\Delta]$, and the current and next data are assigned to $\mathrm{D} 0$ and $\mathrm{D} 1$, respectively; otherwise, Nt is reset to zero. Thirdly, the difference between D0 and D1 in the subsequent time period is further compared with $\Delta$. To prevent the count of run length to be too long to disorder the data structure, the run length is automatically reset after adding to a particular value (e.g. 32767 for a 15-bit run length), and the aforementioned 3-step procedure is then repeated until the sampling is completed. The data of each channel can be compressed in parallel by using the above mentioned procedure.

Table 1 . Telemetry data format.

\begin{tabular}{|c|c|}
\hline D15 & D14 $\sim$ D0 \\
\hline 1 & AD quantized data \\
\hline 0 & Run length (0 to 32767$)$ \\
\hline
\end{tabular}

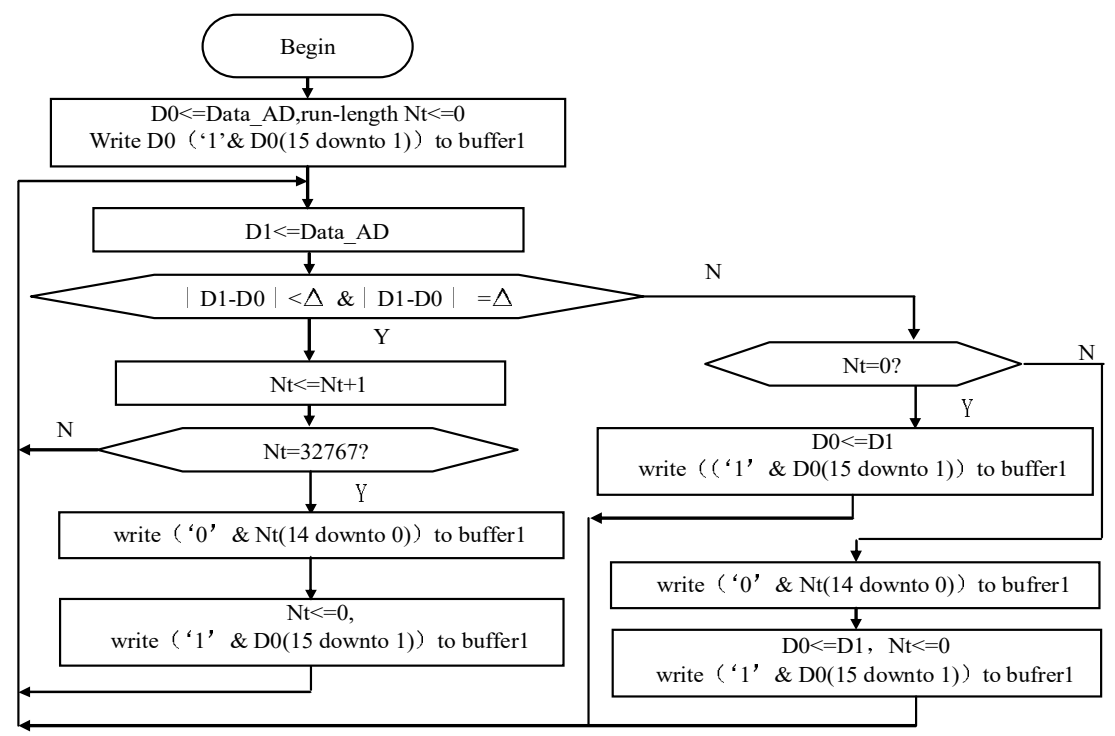

Fig. 3. Flow chart of run-length compression.

\subsection{Two-stage framing}

For the multichannel signals of sensor networks, the correlation of data between adjacent channels was neglected because the oversampling processor was set to the sampling polling mode. A data packet was designed to improve the compression method (Fig. 4). In Fig. 4, CHMN denotes the quantization data of a corresponding channel, $\mathrm{CH}$ NUM is the number of channels, RCX is the row count, FCx is the frame count, RSYNx is the row synchronous word, and FSYNx is the frame synchronization word. The data packet is divided into two stages. On the first level, the sampled data of each channel are compressed and packed into small data packets called sub-frames. Each sub-frame contains labelling, a synchronous word, and 
a channel number and corresponds to one channel, thereby ensuring that all data in the same channel are stored in a sub-frame. Each channel contains one cache, which is used to store the compressed data of a current channel. The total of 16 sub-frames correspond to 16 channels. Therefore, the designed structure can effectively distribute data with high relevance, thereby enhancing the data compression ratio. The data structure is shown in Table 2. RSYNx of the sub-frame is "EB 90". FSYNx of the sub-frame is " $146 \mathrm{~F}$ ". On the second level, the sub-frames are repacked into a new data packet called a prime frame, in which the frame count and frame labelling of every sub-frame are included. When the size of data stored in this cache equals the size of the sub-frame, these data are read out and then written into a buffer. In the buffer, a frame sign, a channel number, and a time count are all included in the new data packet with the primeframe format (Table 3). The frame sign is the beginning of a data frame, and it is used to distinguish between different data frames. The time count labels the time of current data framing and is often regarded as the frame count.

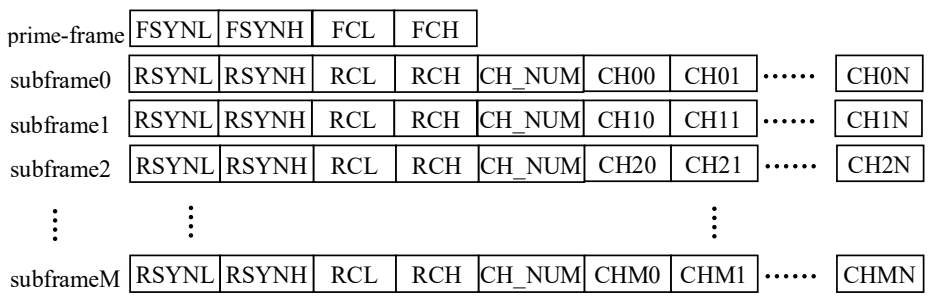

Fig. 4. The two-stage framing format.

Table. 2. The subframe format.

\begin{tabular}{|c|c|c|c|c|c|}
\hline Data 0 & Data 1 & Data 2 & Data 3 & Data 4 & Data 5 to Data 511 \\
\hline EB & 90 & RCL & RCH & CH_NUM & Data \\
\hline
\end{tabular}

Table 3. The prime-frame format.

\begin{tabular}{|c|c|c|c|c|}
\hline Data 0 & Data 1 & Data 2 & Data 3 & Data 4 to Data 8195 \\
\hline 14 & $6 \mathrm{~F}$ & $\mathrm{FCL}$ & $\mathrm{FCH}$ & Data \\
\hline
\end{tabular}

\subsection{Hardware implementation of telemetry data compression}

The acquisition and compression of telemetry data were performed in the multichannel acquisition system, as shown in Fig. 5. The system consists of an input interface, a signal adjustment circuit, an input voltage follower, an analog multiplexer (MUX), an output voltage follower, an A/D converter, a data buffer, and an FPGA. The test data are transmitted through the interface to a signal adjustment module in which the amplitudes of signal are adjusted and high-frequent noise is filtered. Under the control of FPGA, the adjusted signals of specified channels are output through the voltage follower circuit and the multi-channel analogue switch successively and then are converted by the ADC module. Finally, the compression process of test data is implemented in FPGA. The whole internal logic procedure of FPGA is shown in Fig. 6. The telemetry data are oversampled and transmitted into the A/D converter. Then, the arithmetic average in the internal logic of FPGA is implemented to approximate the oversampled data to the actual signal value to achieve reduction of disturbance and error. This part of the internal function of FPGA is called an oversampled filter. Next, the data are compressed using the RLE encoding and stored in corresponding buffers. Finally, the data are packed and written into FIFO, ready for output by sending to the data logic module. 
a)

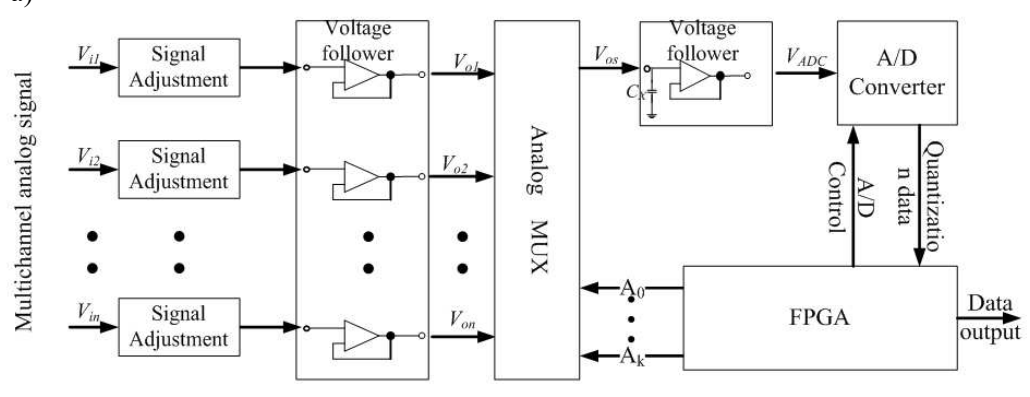

b)

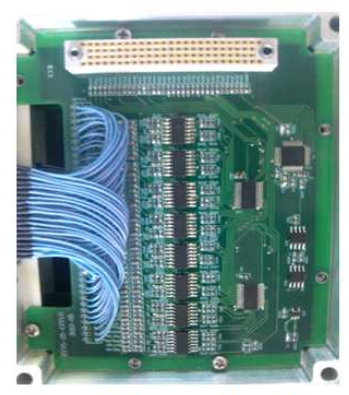

Signal adjustment board

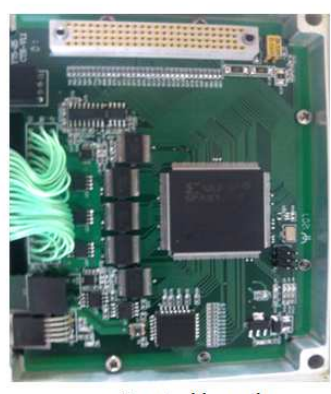

Control board

Fig. 5. A schematic (a) and hardware circuits of the multichannel acquisition system (b).

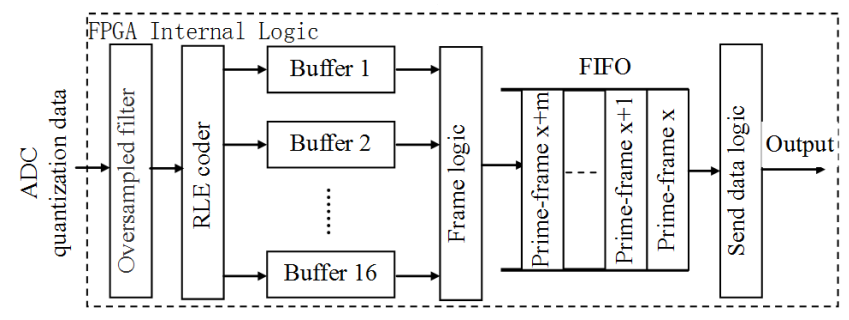

Fig. 6. The internal logic of FPGA.

The proposed approach can achieve the real-time compression of the sampled data. The whole process adopts a pipeline mode. First, the oversampled data are averaged. The data from each channel are then classified based on their frame sign. Then, they are compressed via the run-length encoding method in parallel. Finally, the data of each channel are written into a buffer as a format of the sub-frame. Therefore, the proposed compression method does not cause latency.

\section{Measurement results}

The test telemetry signals used in our study consist mainly of pulse signals and vibration signals collected by shock sensors and vibration sensors throughout repeated measurements, respectively. The method mentioned in [23] and the popular WinRAR method were used as the control groups. Two multichannel acquisition systems with the same structure were used to sample the same signals simultaneously to evaluate the performance of a proposed compression method in terms of compression ratio, distortion degree, and system precision. The proposed compression method was implemented in one acquisition system, and the data compression 
method mentioned in [23] was implemented in the other system. The sampling frequency was $12 \mathrm{kHz}$, and the oversampling frequency was $48 \mathrm{kHz}$. The sampled data were read and decompressed by a home-built PC application software. The data were extracted based on the frame format for each channel. All data were then restored if they were not run-length based on the highest bit of the data. The sizes of the telemetry data after compression at different error parameter $\Delta$ values were measured. The compression ratio and distortion degree of the telemetry data and the precision of the acquisition system for these two types of compression method were calculated using the same hardware implementation platform.

Figure 7 shows that the proposed method significantly (i.e., several-fold) improves the compression ratio compared with the method in [23] for the pulse signal, especially when $\Delta$ is larger than 15 . For example, when the error parameter value is 25 , the compression ratio of the data is 544.46 when the proposed method was used. This value was improved by up to ten-fold. These findings reveal that the compression ratio can be further enhanced by using the proposed method. The enhanced compression ratio is resisted to noise disturbance by averaging the quantization noise.

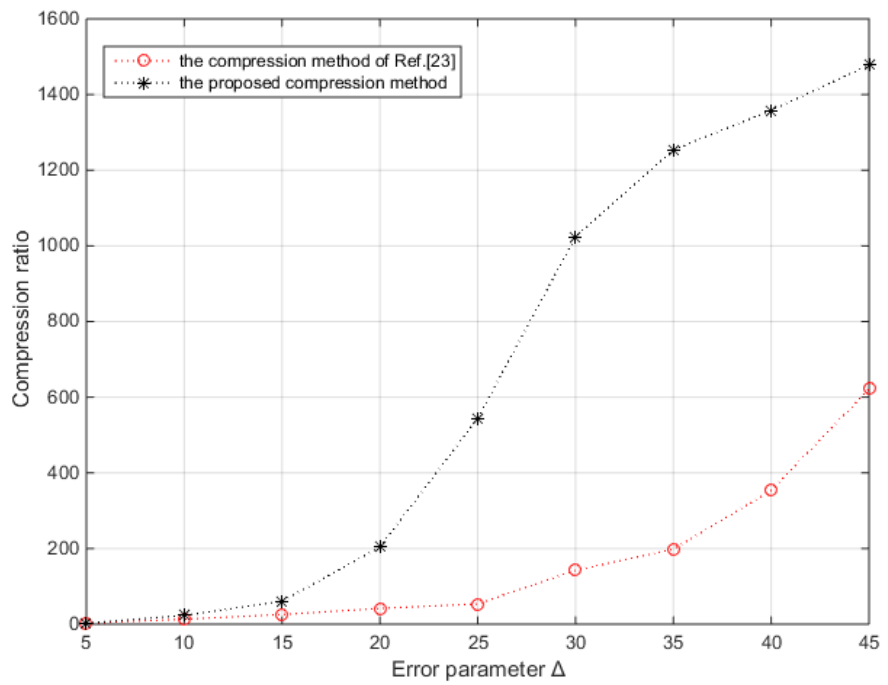

Fig. 7. A relationship between the error parameter and the compression ratio of pulse signals.

Figure 8 shows a comparison between the original waveform and the compressed signal waveform after decompression when the error parameter value is 25 . The original waveforms mentioned in Fig. 8 indicate the output signals of the signal adjustment circuit. The pulse waveform after decompression in the proposed method is in agreement with its original waveform. In our study, the distortion degree is defined as a result of dividing the difference between the values of quantized data before and after the compression by the value of uncompressed data, to quantitatively characterize the compression effect of our method. The distortion degrees of the pulse data at different error parameter $\Delta$ values were then compared for the proposed method and the method in [23], as shown in Table 4. The reduction of the distortion degree ranges from $27.78 \%$ to $38 \%$, which indicates that the proposed method has a lower distortion degree compared with the method in [23]. In order to directly reflect an advantage of a high compression ratio for the same compression quality (namely, the identical distortion degree) of the developed method, Fig. 9 demonstrates that the proposed method could achieve a higher compression ratio with a smaller distortion, which exhibits significant advantages in the telemetry data compression. 


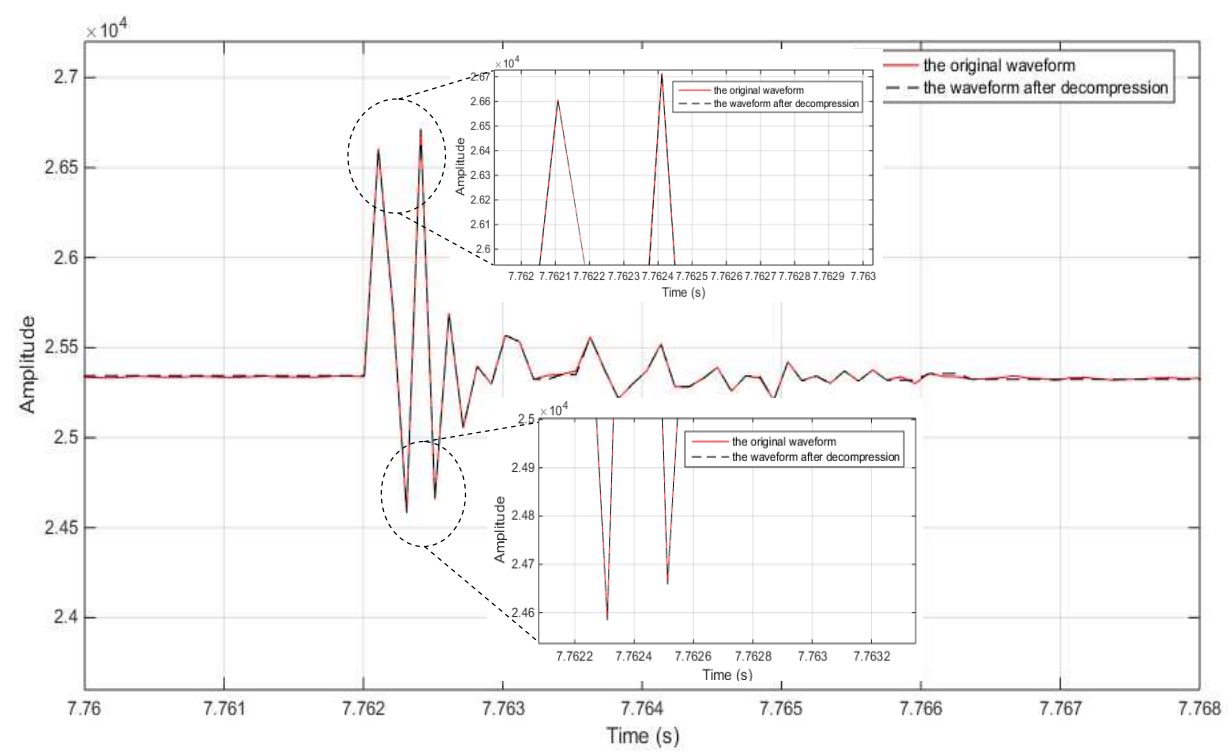

Fig. 8. Comparison of the original waveform with the compressed signal waveform after decompression.

Table 4. Comparison of distortion degrees of the pulse signals.

\begin{tabular}{|c|c|c|c|c|c|}
\hline $\boldsymbol{\Delta}$ & $\mathbf{5}$ & $\mathbf{1 0}$ & $\mathbf{1 5}$ & $\mathbf{2 0}$ & $\mathbf{2 5}$ \\
\hline Method in [23] & $0.027 \%$ & $0.055 \%$ & $0.083 \%$ & $0.11 \%$ & $0.14 \%$ \\
\hline $\begin{array}{c}\text { Proposed } \\
\text { method }\end{array}$ & $0.019 \%$ & $0.039 \%$ & $0.055 \%$ & $0.075 \%$ & $0.087 \%$ \\
\hline Improvement & $29.06 \%$ & $29.12 \%$ & $33.78 \%$ & $31.73 \%$ & $38.00 \%$ \\
\hline$\Delta$ & 30 & 35 & 40 & 45 & \\
\hline Method in [23] & $0.16 \%$ & $0.18 \%$ & $0.21 \%$ & $0.24 \%$ & \\
\hline $\begin{array}{c}\text { Proposed } \\
\text { method }\end{array}$ & $0.11 \%$ & $0.13 \%$ & $0.15 \%$ & $0.15 \%$ & \\
\hline Improvement & $31.25 \%$ & $27.78 \%$ & $28.57 \%$ & $37.50 \%$ & \\
\hline
\end{tabular}

Finally, the precisions of the system when either method was used were compared, as shown in Table 5. The result shows that the proposed method improves the effective number of bits by one bit by adopting the oversampling technology. When the resolution reaches 9.95 bits, the corresponding degree of distortion of the signal is $0.11 \%$, which indicates that the compressed data can be accurately restored in engineering applications.

For multichannel vibration signals, the compression ratios obtained with the proposed method and the method mentioned in [23] are shown in Table 6. The results show that the compression ratio is significantly improved when the proposed method was used, thereby highlighting the effectiveness of our method.

We also compared our method with the WinRAR compression method in terms of the compression ratio, as shown in Table 7 . The WinRAR method is a lossless self-adaptive multiple compression algorithm. The proposed method could be regarded as a lossless compression method when the error parameter is equal to zero. On the contrary, the proposed algorithm works in the loss mode. It is calculated that the compression ratio of the proposed method in the lossless mode is similar to that of the WinRAR method (e.g. 1.8 for the WinRAR 
method and 1.3 for the proposed method for a vibration signal). By adjusting the error parameter (e.g. $\Delta=25$ ), the compression ratio of the proposed method (namely, in the loss mode) is significantly improved. In this situation, the proposed method achieves the trade-off between a low distortion degree (less than $0.1 \%$ ), which meets the practical requirement, and a high compression ratio, compared with WinRAR - the loss method.

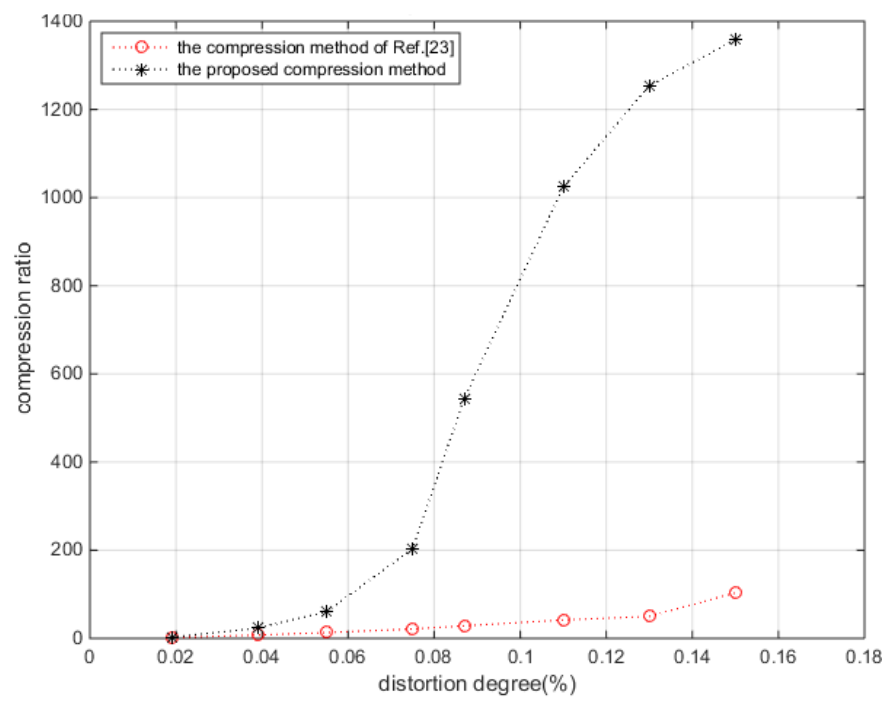

Fig. 9. A relationship between the distortion degree and the compression ratio of pulse signals.

Table 5. Comparison of Resolution (R) and Compression Ratio (CR) of pulse signals.

\begin{tabular}{|c|c|c|c|c|c|c|}
\hline & $\Delta$ & $\mathbf{5}$ & $\mathbf{1 0}$ & $\mathbf{1 5}$ & $\mathbf{2 0}$ & $\mathbf{2 5}$ \\
\hline \multirow{2}{*}{ Method in [23] } & $\mathrm{R}$ (bit) & 9.71 & 9.68 & 9.63 & 9.65 & 9.62 \\
\cline { 2 - 7 } & $\mathrm{CR}$ & 2.63 & 13.23 & 25.72 & 41.77 & 53.51 \\
\hline \multirow{2}{*}{$\begin{array}{c}\text { Proposed } \\
\text { method }\end{array}$} & $\mathrm{R}$ (bit) & 10.75 & 10.71 & 10.68 & 10.65 & 10.61 \\
\cline { 2 - 7 } & $\mathrm{CR}$ & 2.80 & 23.14 & 60.72 & 203.73 & 544.46 \\
\hline & $\Delta$ & 30 & 35 & 40 & 45 & \\
\hline \multirow{2}{*}{\begin{tabular}{c} 
Method in [23] \\
\cline { 1 - 7 }
\end{tabular}} & $\mathrm{R}$ (bit) & 9.59 & 8.96 & 8.92 & 8.85 & \\
\cline { 2 - 7 } $\begin{array}{c}\text { Proposed } \\
\text { method }\end{array}$ & $\mathrm{CR}$ & 142.58 & 198.2 & 353.6 & 621.7 & \\
\cline { 2 - 7 } & $\mathrm{CR}$ & 10.58 & 9.99 & 9.98 & 9.95 & \\
\hline
\end{tabular}

Table 6. The compression ratio of vibration signals.

\begin{tabular}{|c|c|c|c|c|c|}
\hline $\boldsymbol{\Delta}$ & $\mathbf{5}$ & $\mathbf{1 0}$ & $\mathbf{1 5}$ & $\mathbf{2 0}$ & $\mathbf{2 5}$ \\
\hline Method mentioned in [23] & 1.32 & 4.52 & 11.18 & 26.19 & 42.17 \\
\hline Proposed method & 1.58 & 6.28 & 21.85 & 37.49 & 81.92 \\
\hline$\Delta$ & 30 & 35 & 40 & 45 & \\
\hline Method mentioned in [23] & 50.37 & 57.84 & 62.43 & 69.85 & \\
\hline Proposed method & 149.12 & 173.78 & 197.59 & 214.33 & \\
\hline
\end{tabular}


Table 7. Comparison of compression ratios obtained with WinRAR and the proposed method.

\begin{tabular}{|c|c|c|}
\hline Type & WinRAR method & Proposed methoc \\
\hline Pulse signal & 3.92 & $545.8(\Delta=25)$ \\
\hline Vibration signal & 1.88 & $81.92(\Delta=25)$ \\
\hline
\end{tabular}

\section{Discussion}

All telemetry signals of the whole testing process should be sampled and recorded to determine the state of aircraft in the experiment. Fast-varying signals are the main components of total data at high sampling rates, and these signals consist of active and smooth transition phases for the whole test time. The active phase of fast-varying signals in the total flight process is less than $20 \%$, whereas the smooth transition phase accounts for $80 \%$ of the total fast-varying signals $[12,23]$. In the smooth transition phase, the signal values show no significant variations, which can be considered as redundant data. Therefore, the redundant data provide space for data compression. If the data are not preprocessed before the compression encoding, the noise during sampling may increase the difference between adjacent redundant data, which highly affects the compression performance. The oversampling used in this method enables a significant reduction of noise signals in the smooth transition to achieve a high compression ratio in appropriate error parameter conditions. Moreover, the oversampling improves the precision of the sampled data. Thus, the distortion degree and acquisition resolution of the telemetry data are highly improved. Based on these results, the proposed method can further improve the compression performance to meet the requirements in practical engineering applications. Therefore, the proposed method is significantly more effective when applied to high-precision high-capacity acquisition systems, compared with the existing studies.

Developing a data compression method with a high compression performance is necessary to store and transmit large quantities and various types of telemetry data. An enhanced and effective multichannel telemetry data compression method is proposed to balance the compression ratio, precision, and distortion. The proposed approach consists of three modularized phases, as follows: 1) oversampling and normal averaging of the telemetry data, 2) the data compression based on RLE with an error parameter, and 3) two-stage framing. The proposed data compression method is based on FPGAs. The proposed method significantly improves the compression ratio of the telemetry data and the system accuracy as well as reduces the distortion degree of the data, compared with the results of previous research.

The high performance and simple hardware implementation of our proposed method provide an effective data compression application to high-precision multichannel acquisition systems and high real-time testing.

\section{Acknowledgments}

This work was financially supported by the National Natural Science Foundation of China (Grant No.61004127 and No.11502156).

\section{References}

[1] Monteiro, A., Lu, W., Gough, M., Thompson, J., Yearby, K. (1996). A smart telemetry compression system for a space instrument: MARS-96 ELISMA instrument complex. Microprocessors Microsyst, 20(1), 17-30.

[2] Mulholland, J.E., Tamney, F.K., Jr. (1996). Spacecraft telemetry link performance in a transfer orbit. IEEE Transactions on Aerospace and Electronic Systems, 32(4), 1321-1335. 
[3] Drost, G.W., Bourbakis, N.G. (2001). A Hybrid System for Real-time Lossless Image Compression. Microprocessors and Microsystems, 25(1), 19-31.

[4] Lin, M., Lee, J., Jan, G. (2003). A lossless data compression and decompression algorithm and its hardware architecture. IEEE Trans. Very Large Scale Integr. (VLSI) Syst., 11(3), 499-510.

[5] Abo-Zahhad, M., Rajoub, B. (2002). An effective coding technique for the compression of one dimensional signals using wavelet transforms. Medical Engineering and Physics, 24(3), 185-199.

[6] Kattan, A. (2010). Universal intelligent data compression systems: a review. Proc. 2nd Conf. Computer Science and Electronic Engineering Conference (CEEC), Colchester, UK, 11597641, 1-10.

[7] Cristiano, M., Ivanil, A., Bonatti, S., PeresAn, P.L.D. (2013). Adaptive Run Length Encoding method for the compression of electrocardiograms. Medical Engineering \& Physics, 35, 145-153.

[8] David, A., Maluf, P., Tran, B., Tran, D. (2008). Effective Data Representation and Compression in Ground Data Systems. IEEE Aerospace Conference, 1-7.

[9] Qian, S.E., Bergeron, M., Cunningham, I., et al. (2006). Near lossless data compression onboard a hyperspectral satellite. IEEE Transactions on Aerospace and Electronic Systems, 42(3), 851-866.

[10] Kiely, A., Klimesh, M. (2003). The ICER Progressive Wavelet Image Compressor. IPN Progress Report, 42-155.

[11] Logeswaran, R. (2004). Fast Two-Stage Lempel-Ziv Lossless Numeric Telemetry Data Compression Using a Neural Network Predictor. Journal of Universal Computer Science, 10(9), 1199-1211.

[12] Rong, C.D., Hong, L.J., Yong, Z.G. (2001). Algorithm design for launch vehicle telemetry data compression. Journal of Astronautics, 22(2), 12-17.

[13] Chan, H.L., Siao, Y.C., Chen, S.W. (2008). Wavelet-based ECG compression by bit-field preserving and running length encoding. Computer Methods and Programs in Biomedicine, 90(1), 1-8.

[14] Steinwandt, R., Villányi, V.I. (2008). A one-time signature using run-length encoding. Information Processing Letters, 108(4), 179-185.

[15] Stabno, M., Wrembel, R. (2009). RLH: Bitmap compression technique based on run-length and Huffman encoding. Information Systems, 34(4-5), 400-414.

[16] Korpela, E., Forsten, J., Hamalainen, A., et al. (2006). A hardware signal processing platform for sensor systems. IEEE Aerospace and Electronic Systems Magazine, 21(5), 22-25.

[17] Nunez, J.L., Jones, S., (2002). Lossless Data Compression Programmable Hardware for High-Speed Data Networks. Proc. IEEE Int. Conf. on Field-Programmable Technology, 290-293.

[18] Kao, C., Huang, S., Huang, I. (2007). A hardware approach to real-time program trace compression for embedded processors. IEEE Trans. Circuits Syst. I-Regul. Pap., 54(3), 530-543.

[19] Hashempour, H., Lombardi, F. (2005). Application of arithmetic coding to compression of VLSI test data. IEEE Trans. Computers, 54(9), 1166-1177.

[20] Lee, J., Kim, S., Weems, C. (2002). Performance analysis of a selectively compressed memory system. Microprocessors Microsyst, 26(2), 63-76.

[21] Olyaei, A., Genov, R. (2007). Focal-Plane Spatially Oversampling CMOS Image Compression Sensor. IEEE Trans. Circuits Syst. I-Regul. Pap., 54(1), 26-34.

[22] Xu, X., Dong, G., Feng, Y., Xu, S. (2006). A new spaceborne compression approach for remote sensing imagery. Proc. SPIE, 6031.

[23] Ren, Y., Liu, X., Xu, W., Zhang, W. (2008). Multi-Channel Data Compression. IEEE Aerosp. Electron. Syst. Mag., 23(9), 14-21.

[24] Kim, H., Jung, Y., Kim, H., Ahn, J., Park, W., Kang, S. (2010). A high performance network-on-ship scheme using lossless data compression. IEICE Electron. Express, 7(11), 791-796.

[25] Stewart, R.W., Pfann, E. (1998). Oversampling and sigma-delta strategies for data conversion. Electronics \& Communication Engineering Journal, 10(1), 37-47.

[26] Tan, L., Wang, L.M. (2011). Oversampling Technique for Obtaining Higher Order Derivative of LowFrequency Signals, Instrumentation and Measurement. IEEE Transactions, 60(11), 3677-3684.

[27] Temes, G.C., Candy, J.C. (1990). A tutorial discussion of the oversampling method for A/D and D/A conversion, Circuits and Systems. IEEE International Symposium, 2, 910-913.

[28] Nunez, J., Jones, S. (2003). Run-length coding extensions for high performance hardware data compression. IEE Proc.-Comput. Digit. Tech., 150(6), 387-395. 